dinitrate, ISDN) were performed with dual sensor pressureflow wire (ComboWire ${ }^{\circledR}$ ) advanced down coronary artery $(C)$ and were electronically routed into the intracardiac analyser (CD Leycom, Netherlands) via which LV haemodynamics using pressure-volume (PV) loop catheter in LV apex - were measured, thus enabling simultaneous recordings. Coronary blood flow (CBF), wave intensity analysis and LV pressure volume loops were analysed with specially developed in-house software (MatLab R2014b) (E); (paired t-test; linear regression).

Results Accurate delineation and timings of the backward and forward waves were described relative to cardiac contraction and relaxation $(\mathrm{F})$. Backward expansion wave (BEW) occurred during isovolumic relaxation and correlated for the first time with peak dpdt- $\left(\mathrm{r}^{2} 0.4 \mathrm{p}=0.0015\right)$ and found to be load dependent (F); forward compression wave (FCW) occurred immediately following peak dpdt+; ISDN drove leftward, downward shift of PV loop (D) accompanied by two-step cardiovascular response 1) significant afterload reduction (endsystolic pressure $127+/ - \pm 23 ; 98 \pm 22 \mathrm{mmHg} ; \mathrm{p}<0.01)$ associated with reduction in cardiac work (pressure volume area $1.4 \pm 0.4 ; 1.1 \pm 0.4 \mathrm{~J}$ ) total peripheral resistance and arterial elastance representing systemic vasodilation and significant improvement in mechanical efficiency (68 to 74\%) 2) Reduction in preload $(14 \pm 5 ; 10 \pm 5 \mathrm{mmHg} ; \mathrm{p}<0.01)$ was associated with improved passive (EDPVR B) and active (Tau) diastolic relaxation $(38 \pm 4 ; 36 \pm 4 \mathrm{~ms} ; \mathrm{p}<0.01)$. Despite a reduction in perfusion pressure, coronary flow was maintained.

Conclusion For the first time in humans we relate coronary flow to cardiac contraction-relaxation and describe how ISDN exerts an anti-anginal effect by reduction in afterload and preload, therefore cardiac work and improved cardiac efficiency, maintaining coronary flow despite reduction in perfusion pressure, thus vastly increasing our understanding of the integrated cardiovascular response.

\section{A SYSTEMATIC REVIEW OF THE CLINICAL APPLICATIONS OF CARDIOGONIOMETRY IN CARDIOVASCULAR DISEASE}

${ }^{1}$ Oliver Brown*, ${ }^{2}$ Jennifer Rossington, ${ }^{2}$ Andrew Clark, ${ }^{2}$ Angela Hoye. ${ }^{1}$ Hull York Medical School; ${ }^{2}$ Hull and East Yorkshire Hospital NHS Trust; *Presenting Author

\subsection{6/heartinl-2016-309890.95}

Introduction Cardiogoniometry (CGM) is a novel method of 3-D electrocardiographic (ECG) assessment, which has previously been shown to have significant diagnostic accuracy at identifying patients with stable coronary artery disease (CAD) and acute coronary syndromes (ACS). However, there is considerable variation of reported diagnostic accuracy of CGM depending on the gold standard test used to compare it to. The aim of our review is to identify what is diagnostic
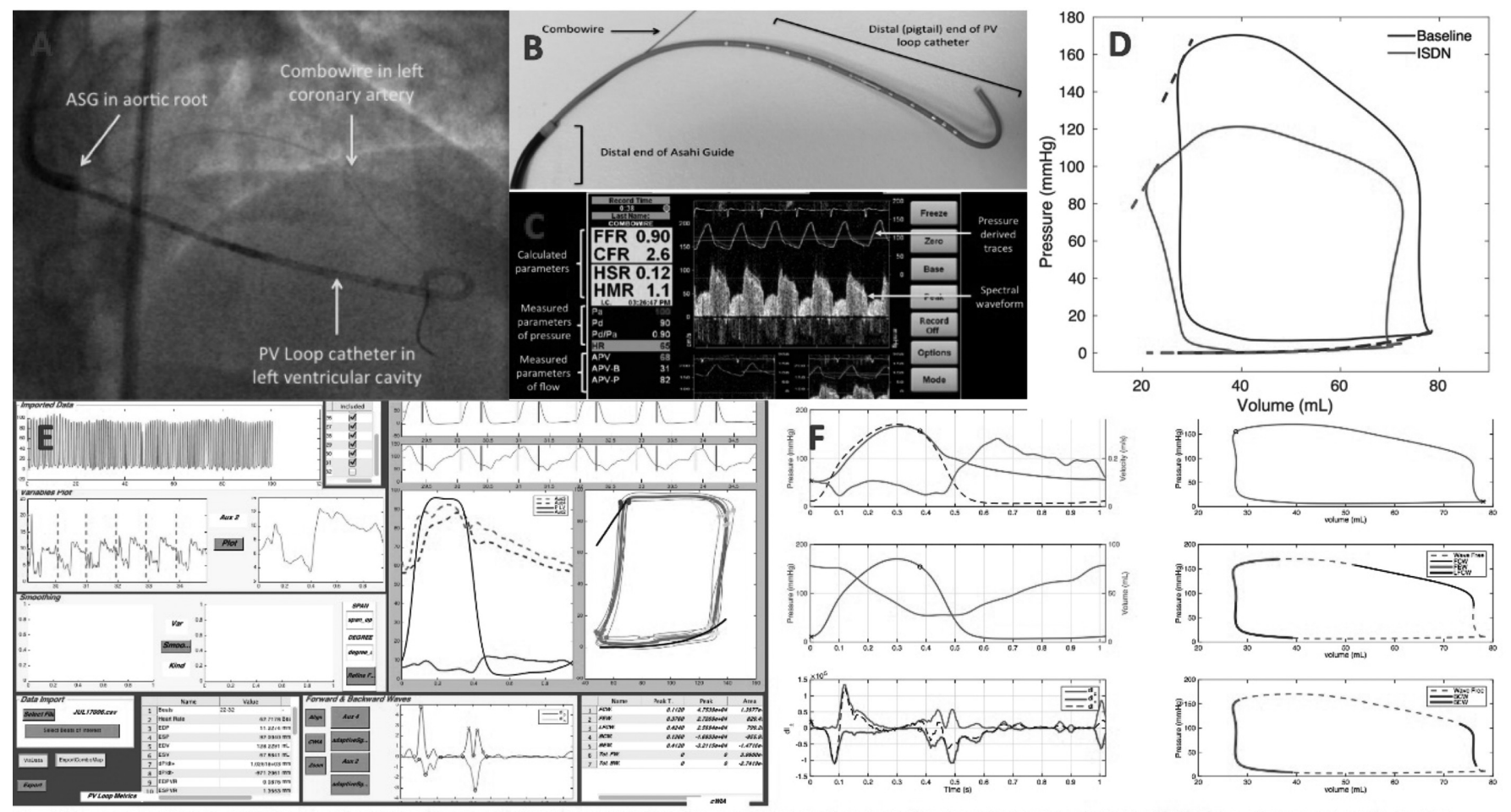

Abstract 94 Figure 1 A. Flouroscopic image of PV loop in the catheter in the LV with combowire in the coronary artery; B. Externalisation of guide catheter with PV loop and pressure-flow wire; C. Coronary flow data obtained on the Combomap console (Volcano Corp); D. LV PV loop at rest (blue) and following administration of nitrates (red) with a reduction in cardiac work; E. In-house softwere developed on MatLab R2014b - snapshot delineating LEFT coronary blood flow, MIDDLE. LV (black), aortic (red) and distal coronary pressure (blue) and RIGHT PV loop. F. Left upper and depicting distal coronary pressure and flow, left middle: LV pressure and volume plotted against time and left lower: wave intensity analysis, right middle forward going waves in coronary circulation depicted in relation to cardiac contraction, right lower: backward travelling (suction) waves in relation to cardiac cycle 
accuracy of CGM at identifying stable CAD and ACS, and identify other novel clinical applications of the technology.

Methods Using a pre-defined search strategy, electronic databases (MEDLINE and Embase) were searched for papers published between 1946-June 2015 and reference lists were pursued. For inclusion, papers had to be original research articles that investigated the clinical application of CGM. Sixteen publications were identified from our search; seven investigated the diagnostic accuracy of CGM to identify stable CAD; one investigated the diagnostic accuracy of CGM to identify ACS; one investigated CGM as a screening tool for cardiac allograft vasculopathy in heart transplant patients and seven investigated specific CGM parameters without commenting on diagnostic accuracy. If data was available and suitable for collation, it was pooled to calculate a comprehensive overview on markers of diagnostic accuracy, which included sensitivity and specificity.

Results When looking at studies investigating the diagnostic accuracy of CGM to identify stable CAD the figures of diagnostic accuracy varied: Sensitivity (64-89\%) and specificity (64\%-82\%), however CGM consistently outperformed 12-lead ECG in terms of sensitivity (29-76\%) and was comparable in terms of specificity (18-95\%). The collated data pooled for our analysis included 772 patients, of which 473 had stable CAD. Our analysis showed CGM has a sensitivity of $70 \%$ and specificity of $82 \%$ at identifying stable CAD, being significantly more accurate than 12-lead ECG. The one study identified investigating the diagnostic accuracy of CGM to identify ACS included patients with non-ST segment elevation ACS and used multiple comparators including 12-lead ECG. It showed that the sensitivity and specificity of CGM to identify nonST segment elevation ACS was 69\% and 54\% respectively, having significantly higher sensitivity than 12-lead ECG. The diagnostic accuracy reported for CGM to detect cardiac allograft vasculopathy was $100 \%$ and $88 \%$ respectively for sensitivity and specificity. Conclusion CGM has superior diagnostic accuracy to 12-lead ECG at identifying patients with stable CAD and is significantly more sensitive than 12-lead ECG at identifying patients with non-ST segment elevation ACS. It may have an important role in screening patients with stable CAD and those with acute chest pain.

\section{THE IMPACT OF "CONSULTANT OF THE WEEK (COW)" INPATIENT MEDICAL CARE ON PATIENT OUTCOMES}

${ }^{1}$ Sophie Gu*, ${ }^{2}$ Michael Kuehl, ${ }^{3}$ Richard Watkin. ${ }^{1}$ North West London NHS; ${ }^{2}$ Queen Elizabeth Hospital Brimingham; ${ }^{3}$ Good Hope Hospital; *Presenting Author

10.1136/heartjnl-2016-309890.96

Introduction There appears to be growing evidence that increasing consultant-led multidisciplinary team (MDT)-delivered care is associated with better patient outcomes, quicker decision making and more efficient uses of resources. There is still inconsistency across different hospitals, on how consultant led care can be best delivered when treating inpatients. Our hypothesis was that compared to a five single on call day service by separate Cardiologists, a consultant-led oncall week which consists of a twice daily inpatient ward round by the same consultant would improve patient outcomes in terms of discharge rates (DRs), length of stay (LoS), inpatient mortality rates (MRs) and readmission rates (RRs).

Settings Good Hope Hospital is a 521 bed district general hospital with 6 consultant cardiologists, and has an inpatient capacity of 6 CCU and 22 cardiology beds. Traditionally, all patients on CCU as
Abstract 96 Table 1 Comparison of monthly patient discharges, length of stay, readmission rate and inpatient mortality prior to and after the intervention

\begin{tabular}{llll}
\hline Variable & $\begin{array}{l}\text { Monthly mean (April 2012 } \\
\text { to November 2013) }\end{array}$ & $\begin{array}{l}\text { Monthly mean (December } \\
\text { 2013 to February 2015) }\end{array}$ & $\begin{array}{l}\mathbf{P} \\
\text { value }\end{array}$ \\
\hline $\begin{array}{l}\text { Discharged } \\
\text { patients ( } \mathrm{n})\end{array}$ & $103 \pm 12$ & $118 \pm 17$ & $\mathrm{P}=$ \\
$\begin{array}{l}\text { Length of } \\
\text { stay (days) }\end{array}$ & $7.9 \pm 1.0$ & $6.4 \pm 1.0$ & 0.008 \\
$\begin{array}{l}\text { Readmission } \\
\text { rate (\%) }\end{array}$ & $21.7 \pm 7.9 \%$ & $23.4 \pm 6.6 \%$ & $\mathrm{P}=$ \\
Inpatient & $4.3 \pm 1.6 \%$ & & 0.0001 \\
Mortality (\%) & & $4.2 \pm 1.5 \%$ & $\mathrm{P}=$ \\
\hline
\end{tabular}

well as new patients on the ward were reviewed by the on call consultant who was on call on a set day of the week. After the initial post take ward round, these patients were seen on a twice weekly basis by their admitting consultants and were looked after by junior doctors In the interim.

Methods This was a retrospective observational study of inpatient average length of stay and discharge rates between April 2012 to March 2015 and included the data of 3289 patients. The intervention was implemented on $1^{\text {st }}$ November 2013. All data were collected by the hospital IT department on a daily basis and reported on a monthly basis. We compared the inpatient MRs and RRs to assess any adverse effects on the quality of patient care. Statistical analysis was performed using student T-test. The $\mathrm{p}$ value $<0.005$ was considered significant. Results are expressed as means \pm SD.

Results The data of 2058 patients prior to the consultants of the week method were compared to 1771 after the change. The monthly means \pm SD of discharge rates, length of stay, readmission rate and mortality rate are shown in table 1 . There is a significant increase in discharges and reduction in length of stay following the intervention. Despite a $15 \%$ increase in patient discharge rate, the readmission rate and inpatient mortality rate did not change significantly.

Conclusions Focused daily consultant input has a significant impact on reducing inpatient length of stay, ensuring timely discharges, and saving the NHS resources in bed days and creating more beds available for new admissions.

\section{Acute coronary syndromes}

\section{MULTI-VESSEL ANGIOPLASTY AT THE TIME OF STEMI HAS EQUIVALENT MORTALITY TO A CULPRIT ONLY STRATEGY: RESOLVING THE PARADOX OF RANDOMISED CONTROLLED TRIALS AND OBSERVATIONAL STUDIES IN MULTIVESSEL DISEASE AND STEMI}

Yousif Ahmad*, Chris Cook, Ricardo Petraco, Sukhjinder Nijjer, Rasha Al-Lamee, Matthew Shun-Shin, Daniel Keene, Ashwin Balu, Iqbal Malik, Christopher Baker, Ghada Mikhail, Amarjit Sethi, Rodney Foale, Justin Davies, Jamil Mayet, Darrel Francis, Sayan Sen. Imperial College London; *Presenting Author

10.1136/heartjnl-2016-309890.97 